\title{
Animal voice recognition for identification (ID) detection system
}

\begin{abstract}
Voice recognition systems have become the important applications for speech recognition technology. In this paper, an animal identification (ID) detection system based on animal voice pattern recognition algorithm has been developed. The developed animal voice recognition system uses the zero-cross-rate (ZCR), Mel-Frequency Cepstral Coefficients (MFCC) and Dynamic Time Warping (DTW) joint algorithms as the tools for recognizing the voice of the particular animal. ZCR is used for the end point detection of input voice such that the silence voice can be removed. MFCC is used for the process of feature extraction where a more compact and less redundant of the representative voice can be obtained from the input voice. While the voice pattern classification will be done by using DTW algorithm. The DTW voice pattern classification module is playing a very important role as it is used to get the optimal path between the input voice and the reference voice in the database. The obtained results show that the developed recognition system can be worked as expected.
\end{abstract}

Keyword: Animal; DTW; MFCC; Voice recognition; ZCR 